\title{
A case of fatal pressure on the neck in a woman with a stab injury
}

Kitulwatte IDG ${ }^{1}$, Pollanen $M S^{2}$

${ }^{1}$ Department of Forensic Medicine, Faculty of Medicine, Ragama

${ }^{2}$ Forensic Pathology Unit, Office of the Chief Coroner for Ontario, Canada

*Corresponding author: Tel: 2958219. E-mail address: indiradgk@yahoo.com

\begin{abstract}
Evidence of pressure on the neck forms a spectrum of degree from minimal to marked. We report a case of fatal pressure on the neck with minimal external signs. A 27 year old woman was found dead in her home. A blood stained knife and a blood stained bathrobe belt with hair entangled in it were discovered at the scene. Autopsy revealed a stab injury to the chest extending to the right lung and multiple contusions to the head, face and extremities. There were petechial haemorrhages on bilateral eyelids and conjunctivae. There were 2 small contusions on the front of the neck. Special neck dissection revealed a small haemorrhage in the sternocleidomastoid muscle and a haemorrhagic fracture of the right superior horn of the thyroid cartilage. Cause of death was given as pressure on the neck in a woman with stab injury to the right chest. Petechial haemorrhages play a major role in confirming the cause of death when there is minimal evidence for application of pressure on the neck.
\end{abstract}

Keywords : Pressure on the neck, stab injury, minimal signs, fracture superior horn, petechial haemorrhages

\section{Introduction}

Evidence of pressure on the neck forms a spectrum of degree from minimal to marked. There is no agreement for the required minimal number and nature of lesions to make the diagnosis of strangulation. [1, 2, 3] Petechial haemorrhages though not specific for pressure on the neck or asphyxia are important indicators for such mechanisms. [4]

\section{Case report}

A 27 year old female was discovered dead on the floor of her bedroom. The bed cover was stained with blood. There was a blood stained bathrobe belt with hair entangled on it and a blood stained knife at the scene. At autopsy, she was a well built young female with petechiae over bilateral eyelids and in the left conjunctivae. (Figure: 3 ) There was a confluent haemorrhage in the right conjunctivae. There were blood stains on the soles of her feet. There was a diagonally oriented stab wound to the right upper back of the chest, measuring $1.8 \mathrm{~cm}$ when approximated. The reapproximated wound demonstrated a blunt corner infero medially and a sharp corner supero laterally. The haemorrhagic stab wound had penetrated the lower lobe of the right lung to a depth of $1 \mathrm{~cm}$. As a result there was $400 \mathrm{ml}$ of haemothorax in the right pleural cavity. There were 2 small blue, linear contusions on the front of the neck with two underlying small haemorrhages in the posterior aspect of the right sterno hyoid muscle and in the posterior belly of the left sternocleido mastoid muscle. There was a fracture, associated with haemorrhage in the base of the right superior horn of the thyroid cartilage. (Figure: 1 \&2) There were multiple small contusions to the head, face and extremities specially the forearms and hands. 


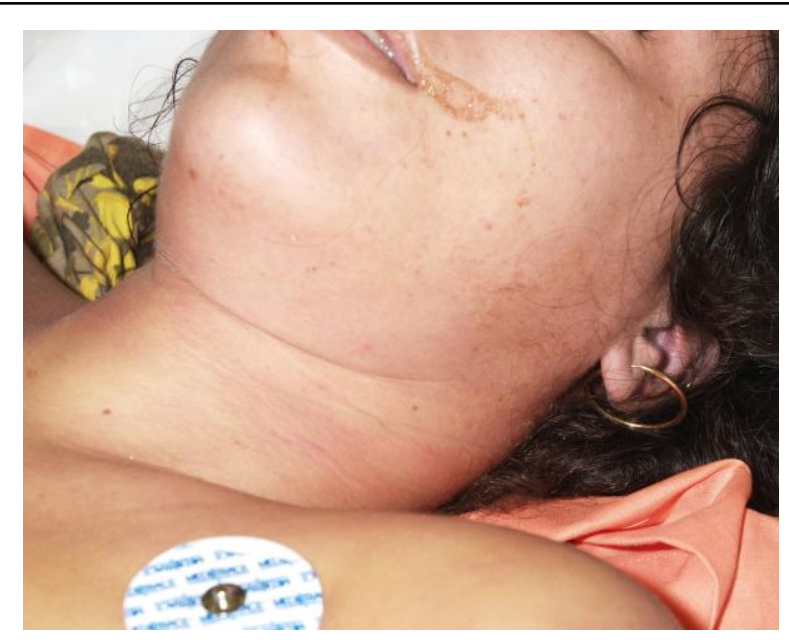

Figure: 1

Front of the neck and face with minimal external iniuries and facial netechial

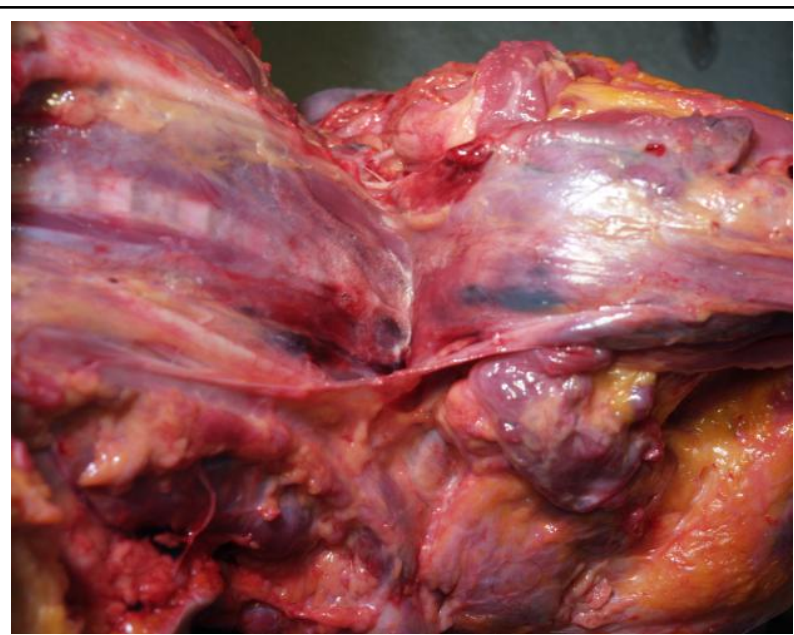

Figure: 2

Haemorrhagic fracture of the right sunerior horn of the thvroid cartilage

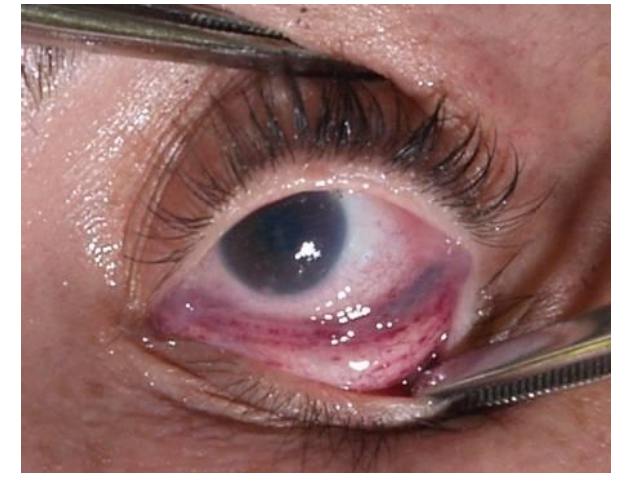

\section{Discussion}

The most interesting finding of this woman was the presence of petechial haemorrhages. They can be observed in almost all cases of fatal neck compression especially in cases of ligature and manual strangulation [5]. The mechanism of formation is increased venous pressure in the head following compression of the veins while the arterial vessels are not totally obstructed leading to rupture of small blood vessels [4]. These areconsidered as 'classic' signs of asphyxia when found together with injuries to the neck. [6].

The deceased had minor external and neck muscle injuries and a fracture in the base of the right superior horn of the thyroid cartilage.
Figure: 3

Petichial haemorrhages in the left conjunctivae
When combined together with the petechial haemorrhages these findings are adequate to make the diagnosis of pressure on the neck [ 1 , 7].

However the mode of application of pressure is difficult to determine. When a soft ligature like a cloth is used the external mark may be incomplete or poorly defined [8]. The extent of injury in ligature strangulation is dependent on the width and elasticity of the ligature and the force applied [9]. There is a higher chance of getting fractures in the hyoid laryngeal complex in manual strangulation $[2,5]$ On the other hand fractures of the horns of the thyroid cartilage were found to be common in ligature strangulation[9]. The presence of the blood stained bath-robe belt with hair entangled in it is a helpful circumstantial evidence to suggest 
ligature strangulation. However from the pathological evidence alone it is hard to conclude.

This woman also had a penetrating injury to the chest with only a small peripheral injury to the lung which bleeds slowly. There was $400 \mathrm{ml}$ of blood within the chest cavity and bloodstains were found on the bed covers and her soles indicating movements. Thus, stabbing was concluded as an ante mortem event. Similarly haemorrhagic neck injuries and the petechial haemorrhages favour pressure on the neck as an ante mortem event. Furthermore in application of pressure on the neck, death occurs in few seconds if both carotid arteries are compressed and in about a minute if only the jugulars are compressed [10]. Therefore, the death should be rapid following the application of pressure on the neck and can be concluded as the cause of death and final event. However the pulmonary blood loss also might have contributed to the death.

The other injuries were mainly contusions located in the forearms indicating self defense [11].

Cause of death was given as pressure on the neck in a woman with stab injury to the right chest. This case illustrates, the difficulties associated with concluding pressure on the neck and highlights the important pathological clues to consider in coming to such conclusion.

\section{Acknowledgements}

The invaluable support given by Miguel Arias and David Clutterbuck of Forensic Pathology Unit, Office of the Chief Coroner for Ontario in obtaining photographs is immensely appreciated.

\section{References}

1. Michael S. Pollanen, A Triad of Laryngeal Hemorrhages in Strangulation: A Report of Eight Cases, J Forensic Sci. 2000 May;45(3):614-8
2. Michael S. Pollanen, and David A. Chiasson, Fracture of the Hyoid Bone in Strangulation: Comparison of Fractured and Unfractured Hyoids from Victims of Strangulation, Journal of Forensic Sciences, JFSCA, Vol. 41, No. 1, January 1996, p. 110-113.

3. Michael S. Pollanen, 1 Barbara Bulger, R.T.(R), 2 and David A. Chiasson, M.D. 1'2 The Location of Hyoid Fractures in Strangulation Revealed by Xeroradiography, Journal of Forensic Sciences, JFSCA, Vol. 40, No. 2, March 1995, pp. 303-305.

4. Susan F. Ely,1 M.D., M.P.H. and Charles S. Hirsch,1 M.D. Asphyxial Deaths and Petechiae: A Review, J Forensic Sci 2000;45(6):1274 -1277.

5. DiMaio, Vincent J. M. M.D. Homicidal Asphyxia; The American Journal of Forensic Medicine \& Pathology. 2000; 21(1):1-4,

6. Michael S. Pollanen, D. Noel McAuliffe Intracartilaginous laryngeal haemorrhages and strangulation; Forensic Science International, 1998; 93 13-20

7. Pollanen M.S, Subtle fatal manual neck compression. Med Sci Law. 2001; 41(2): 13540

8. H. Maxeiner, Britta Bockholdt, Homicidal and suicidal ligature strangulation-a comparison of the post-mortem findings, Forensic Science International 137 (2003) 60-66

9. M.A. Green, Morbid anatomical findings in strangulation, Forensic Sci. 2 (1973) 317-323.

10. David Dolinak, Evan W. Matshes, - Hanging and Strangulation; Forensic Pathology: Principles and Practice Elsevier, Academic Press; 2005; pp209-222

11. Manoj Kumar, Mohanty M, Manoj Kumar Panigrahi, Sachidananda Mohanty, Jyotin Kumar Dash, Shreemanta Kumar Dash; Selfdefense injuries in homicidal deaths; Journal of Forensic and Legal Medicine (2007)14 213 .215

\section{Contribution of authors}

Performing the autopsy-IDGK

Supervision to the autopsy- MSP

Opinion- IDGK, MSP 